\title{
Chateaubriand réviseur et annotateur de ses œuvres, sous la direction de Patrizio TUCCI
}

\section{Laura Brignoli}

\section{OpenEdition}

\section{Journals}

\section{Edizione digitale}

URL: https://journals.openedition.org/studifrancesi/11822

DOI: 10.4000/studifrancesi. 11822

ISSN: 2427-5856

\section{Editore}

Rosenberg \& Sellier

\section{Edizione cartacea}

Data di pubblicazione: 1 avril 2018

Paginazione: 143-144

ISSN: 0039-2944

\section{Notizia bibliografica digitale}

Laura Brignoli, «Chateaubriand réviseur et annotateur de ses œuvres, sous la direction de patrizio Tuccl», Studi Francesi [Online], 184 (LXII | I) | 2018, online dal 03 juillet 2018, consultato il 16 novembre 2021. URL: http://journals.openedition.org/studifrancesi/11822 ; DOI: https://doi.org/10.4000/studifrancesi. 11822

Questo documento è stato generato automaticamente il 16 novembre 2021.

\section{(c) $($ ) $(9)$}

Studi Francesi è distribuita con Licenza Creative Commons Attribuzione - Non commerciale - Non opere derivate 4.0 Internazionale. 


\title{
Chateaubriand réviseur et annotateur de ses œuvres, sous la direction de Patrizio TUCCI
}

\author{
Laura Brignoli
}

\section{NOTIZIA}

Chateaubriand réviseur et annotateur de ses œuvres, sous la direction de Patrizio TUCCI, Paris, Honoré Champion, 2016, 266 pp.

1 Il volume, che raccoglie gli atti del convegno svoltosi a Padova nel dicembre 2007, è suddiviso in tre sezioni (I. «Reprises et remplois», II. «Palimpsestes», III. «Le contrepoint des notes») che richiamano solo in parte la ripartizione delle relazioni proposta nelle tre giornate del convegno. Dalla lettura della prima sezione emerge che la ripresa da un testo all'altro dei «brani», metafora ormai lessicalizzata di cui non si percepisce più la violenza, imponga piuttosto di definirli come «passaggi», mettendo l'accento proprio sull'azione di ciò che passa, e transitando cambia.

2 Così Claude BERCHET (Le dernier avatar des "Geuvres complètes": les "Mémoires d'OutreTombe", pp. 17-33), osservando la stretta relazione fra la vita e la produzione di questo autore che ebbe cura di non smentirsi, insiste sull'immagine di un'opera in costante movimento, mai chiusa su se stessa. La critica passata spesso ha insistito sull'intenzione stilistica delle varianti nei passaggi riutilizzati, ma gli studi di questa sezione mostrano l'insufficienza della spiegazione privilegiata fino ad oggi: Piero tOFFAno (Retour sur les réécritures de la "Nuit chez les sauvages", pp. 35-45) riprende tre versioni della celebre notte e prova in maniera convincente come le varianti inserite nel testo iniziale rispondano non solo a una ideale armonizzazione al contenuto della nuova opera, ma anche a un'esigenza «ideologica». L'operazione è ancora più evidente nel caso di testi originariamente destinati alla stampa periodica che, una volta trasposti nell'opera letteraria, abbandonano il tono cronachistico per inglobare elementi di eternità: il 
romanziere e il cronista si combinano senza mai confondersi (Jean-Marie RoULIN, Le remploi des articles de presse: vers une écriture de l'actualité?, pp. 65-74). Anche quando lo studio è esplicitamente stilistico, come quello di Alain GUYOT (Du "Génie" primitif à "Atala": quelques réflexions sur les écritures de la «matière américaine», pp.47-64) si evidenzia come le preoccupazioni di Chateaubriand non fossero legate solo all'armonia della frase, ma coinvolgessero l'ideologia espressa, la conformità dei generi, l'attenzione alle abitudini letterarie della sua epoca. Jean-Paul cLÉMENT ("De Buonaparte et des Bourbons" et les "Réflexions politiques": Chateaubriand réviseur de ses cuvres politiques, pp. 75-88) analizza la posizione più che le idee politiche di Chateaubriand, e afferma che fu fedele alle sue idee ma senza rinunciare alla duttilità indispensabile alla comprensione profonda della vita che è trasformazione costante. Per questo fu incompreso dai suoi contemporanei che lo accusarono di opportunismo. Infine, partendo da una variante apparentemente insignificante (la scomparsa di un aggettivo dai Mémoires d'Outre-Tombe in un brano ripreso dall'Essai sur la littérature anglaise), JeanChristophe CAVALLIN ("Mémoires" de 1834 et "Mémoires" de 1847: «deux [flamboyants] grands météores», pp. 89-101) ci guida in un vasto tour che si dipana fra la letteratura e la politica, fra Romanticismo e Medioevo, fra le torri del palazzo di Westminster e i sotterranei dell'omonima cattedrale.

3 Molti studiosi sono impegnati nell'edizione critica delle opere complete di Chateaubriand, alcune delle quali ormai pubblicate. È questo il caso dell'Essai sur les révolutions (uscito nel 2009) che, come mostra bene Aurelio PRINCIPATO nel primo saggio della sezione II (La révision inachevée de l'“Essai sur les Révolutions", pp. 105-116), deve più alla scrittura accurata del letterato che all'analisi rigorosa dello storico. Lo studio minuzioso delle varianti comporta spesso una rimodulazione dell'idea dell'opera. Fabienne BERCEGOL (L'enseignement des variantes dans "Atala", pp.117-138) rilegge le varianti di Atala riflettendo su ciò che le ha motivate. Se da una parte le critiche segnalate dai recensori dell'epoca hanno spinto Chateaubriand a intervenire rendendo più fluide le frasi, eliminando le aggettivazioni inutili, correggendo le espressioni enfatiche, attenuando le scene di violenza, dall'altra non accetta di modificare le immagini che più gli stanno a cuore, anche se dovevano piacere poco ai suoi censori. Anche nel Génie du Christianisme Chateaubriand asseconda i critici correggendo le espressioni giudicate troppo audaci o pompose. Gli interventi più importanti, osserva Emmanuelle TABET (Les versions successives du "Génie"; évolution d'une pensée et d'un style, pp. 139-154), riguardano l'uso e la posizione degli aggettivi; modificandoli, si interviene sulla dimensione soggettiva e insieme epica del testo: la sensibilità romantica, con i suoi slanci epici, gli afflati affettivi e lirici ne risulta attenuata. L'opera diventa così più accessibile ai contemporanei e ricondotta a una dimensione argomentativa a discapito della forza poetica del testo, che ne conserva tuttavia qualche traccia. Le correzioni finiscono dunque per influire sul genere dell'opera. Patrizio TUCCI (Les corrections autographes de la Quatrième partie des "Mémoires d'Outre-Tombe" dans le manuscrit de 1845, pp. 155-178) mostra la necessità dell'edizione critica dei Mémoires e analizza non solo il senso delle correzioni introdotte, ma anche il modo in cui esse vennero considerate nelle edizioni critiche precedenti, pur incomplete. Ne scaturisce fra l'altro la determinazione di Chateaubriand a smarcarsi dall'esempio di Rousseau, tanto sul piano personale che in ambito politico. L'analisi grafologica (E. BERVILLÉ, Les autocorrections $d u$ mémorialiste: approche graphologique, pp.179-190), se da una parte conferma tratti 
caratteriali già palesi dalle analisi stilistiche, dall'altra aggiunge delle ipotesi sulle motivazioni inconsce di Chateaubriand.

4 I saggi contenuti nell'ultima sezione, tranne quello di Marika PIVA (Dans les marges, en bas, à la fin: «des notes, toujours des notes», pp. 193-216), che analizza minuziosamente le varie tipologie di note presenti nei testi, si concentrano sulle istanze discorsive. Philippe ANTOINE (Fiction et récit de voyage. Les «Remarques» des "Martyrs", pp. 217-228) analizza il rapporto fra l'Itinéraire e i Martyrs mostrando in quale misura il poeta sa capitalizzare le virtù dello storico e quest'ultimo avvalersi delle tecniche della narrazione. Sul primo di questi due testi riflette anche Elisa GREGORI (Les notes de l' "Itinéraire de Paris à Jérusalem", pp. 229-240), differenziando le diverse istanze che vi intervengono e vanno a costituire un'opera composita in cui convergono elementi autobiografici, finzione narrativa ed erudizione saggistica. Di diffrazione dell'istanza discorsiva parla anche Ivanna Rosi (Chateaubriand juge de François: les notes de 1826 à l'“Essai sur les Révolutions", pp. 241-254), che mette a confronto il giovane François con lo Chateaubriand grande autore e uomo pubblico conscio della sua posizione.

5 A margine, non si può fare a meno di chiedersi se l'ampio scarto temporale fra il convegno e la pubblicazione degli atti abbia indotto alcuni studiosi a una «riscrittura» almeno parziale dei loro testi. Sarebbe stata un'operazione in pieno spirito chateaubriantesco. 